\title{
COLOR IMAGE DEHAZING USING THE NEAR-INFRARED
}

\author{
Lex Schaul, Clément Fredembach, and Sabine Süsstrunk \\ Ecole Polytechnique Fédérale de Lausanne (EPFL) \\ School of Computer and Communication Sciences \\ CH-1015 Lausanne, Switzerland
}

\begin{abstract}
In landscape photography, distant objects often appear blurred with a blue color cast, a degradation caused by atmospheric haze. To enhance image contrast, pleasantness and information content, dehazing can be performed.

We propose that fusing a visible and an near-infrared (NIR) image of the same scene results in a dehazed color image without the need for haze or airlight detection or the generation of depth maps. This is achieved through a multiresolution approach using edge-preserving filtering to minimize artifacts.

The near-infrared part of the spectrum is easy to acquire with normal digital cameras. The NIR images are generally devoid of haze as it is an inherent function of the wavelengths. Experiments on real images validate our approach.
\end{abstract}

Index Terms - Haze, Scattering, Near-infrared, Image fusion, Edge-preserving filters, Multi-resolution

\section{INTRODUCTION}

Images are often degraded by atmospheric haze, a phenomenon due to the particles in the air that scatter light. The intensity of the scattered light is related to that of the incident light by two variables: the photon's wavelength $\lambda$ and the scattering particle's size. Haze is formed when the aerosol particles are smaller than $\lambda / 10$ and the scattering follows Rayleigh's law:

$$
E_{s} \propto \frac{E_{0}}{\lambda^{4}}
$$

i.e., the intensity of the scattered light $E_{s}$ is proportional to that of the incident light $E_{0}$ by the inverse of the fourth power of the wavelength $\lambda$.

Sky and haze appear blue because blue wavelengths are the shortest of the visible spectrum and, therefore, the most scattered. For mist, fog and other weather conditions, caused by particles of larger size, the scattering follows Mie's law and is independent of the wavelength, which is why clouds are achromatic.

Haze causes a loss of contrast and detail as the scattering has an attenuation and smoothing effect. It is particularly problematic in landscape or aerial photography because the effect increases with the distance of the object to the observer, as shown in Fig. 1.

Due to Rayleigh's law (Eq. 1), scattering is significantly smaller in near-infrared (NIR) than in visible images because NIR wavelengths are longer. Haze is much less present, also illustrated in Fig. 1 .

The work presented in this paper was supported by the Swiss National Science Foundation under grant number 200021-124796/1.

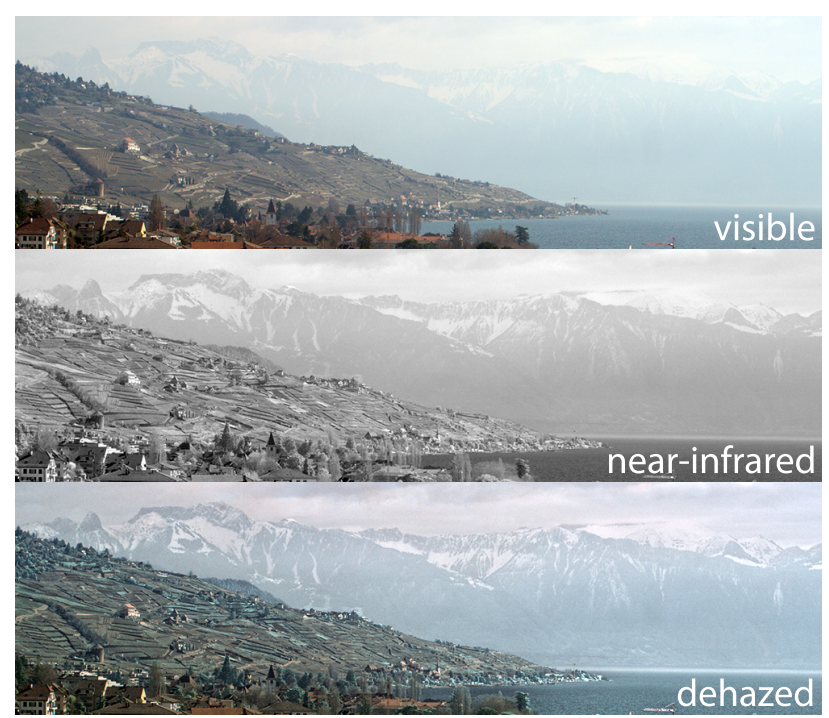

Fig. 1. Example of a scene containing haze. Note the higher contrast and sharpness in the near-infrared image and the dehazed image using our method compared to the visible image.

As opposed to the human visual system, silicon is sensitive to the visible part of the spectrum, ranging from $400 \mathrm{~nm}$ to $700 \mathrm{~nm}$, as well as to the NIR region, from 700-1100 nm. By removing the hot mirror, digital cameras can be enabled to capture visible and NIR images [1].

We propose a method to dehaze images using both visible and NIR images. We apply an edge-preserving multiresolution decomposition based on the Weighted Least Squares (WLS) optimization framework, as described by Farbman et al. [2], to both the visible and the NIR images. We use a pixel level fusion criterion that maximizes contrast. Regions containing haze are thus improved.

The advantage of our approach to dehazing is that no scattering model is required. Multiresolution decompositions using WLS filters are fast and yield few halo artifacts. Regions or images without haze remain unaltered. Therefore, our method can be applied whether haze is actually present or not.

\section{BACKGROUND}

\subsection{Haze Detection and Removal}

Haze induces a loss of contrast, its visual effect is blurring of distant objects. General contrast enhancement approaches can be applied 
for image dehazing, such as linear or gamma correction, histogram stretching/equalization, or unsharp-masking.

As haze is not constant over an image, these techniques cannot be applied globally as they would degrade haze-free regions. Local contrast enhancement techniques are more adapted but require a haze detector, such as Groszek and Allebach's [3] horizon detection.

Photographers use polarization filters to remove the effects of haze because the airlight is (partially) polarized and the light coming from the object of interest is (partially) unpolarized. Schechner et al. [4] improve this approach using different images with different polarization filter orientations.

Usually, however, haze detection and removal go together and are based on atmospheric scattering models. Older approaches need multiple images under different weather conditions [5]. Most singleimage algorithms need additional depth information, e.g., by making use of 3D models [6] or user interaction [7].

Recently, three approaches have been proposed that achieve automatic haze removal using a single image. They make use of the haze imaging equation, which is the sum of two terms, direct attenuation and airlight. Direct attenuation descibes the scene radiance and its decay in the medium, airlight is the ambient light reflected into the line of sight by atmospheric particles [8]. As the problem is underconstrained, correct assumptions need to be made in order to obtain good results.

Tan [9] observes that haze-free images have larger local contrast and that the airlight is smooth. His results, after maximizing local contrast, tend to be oversaturated and can yield halo artifacts. Fattal [10] obtains, after assuming that the transmission and surface shading are uncorrelated, physically correct dehazed images, but his assumption might fail in cases of very dense haze. He et al. [8] introduce the very simple and elegant dark channel prior, based on the observation that very dark pixels exist in natural scenes. The additive airlight brightens these dark pixels, increasing with distance. A depth map can thus be obtained, which is then used to recover the scene radiance.

The disadvantage of these proposed physics based techniques is their complexity. Our method is fast and does not need heuristics for haze and airlight detection.

\subsection{NIR Acquisition}

Camera sensors are made of silicon, sensitive to radiation from 200 $\mathrm{nm}$ to $1100 \mathrm{~nm}$. The $400-700 \mathrm{~nm}$ range is the visible band while the 700-1100 range belongs to the NIR band. The ultraviolet part of the spectrum $(200-400 \mathrm{~nm})$ is generally filtered out by the camera's optical elements. A filter, called hot mirror, protects the sensors from incoming NIR light. By replacing the hot mirror by a piece of glass, the camera is enabled to capture both visible and NIR light [1].

For now, NIR and visible images of the same scene are obtained by placing alternatively a NIR or visible blocking filter on the lens. We are currently investigating a camera design that can simoultaneously capture visible and NIR [11].

\subsection{Multiresulution Image Fusion}

Image fusion is the process of combining information from two or more images of a scene into a single composite image. The images often originate from different sensor responses, for example, visible and thermal-IR data. Image fusion techniques can be categorized into pixel level, feature level, and high level techniques [12].

Multiresolution analysis is a popular technique for pixel level fusion. An image contains important structure at different resolutions.
It can therefore be decomposed into a multiresolution representation where each component captures information present at a given scale, analog to the perception of the human visual system and the composition of real objects. Multiresolution fusion can be split into three steps:

The analysis decomposes an input image $I_{0}$ using spatial filtering into a multiresolution representation composed of approximation images $I_{k}^{a}$ and detail images $I_{k}^{d}$ at different levels $k$. The total number of levels is denoted by $n$. The fusion is then applied pixel-wise at each level $k$. For each image $I_{k}^{a}$ and $I_{k}^{d}$, one of their pixels is chosen according to a criterion, e.g., maximum, minimum or average. The criterion is application-dependent. The inverse transform of the analysis is the synthesis, where the original image is reconstructed from the multiresolution representation.

Many different techniques exist based on pyramid schemes or wavelets, the latter having the advantage of not containing redundant information. For a more complete survey, we refer to [13].

\subsection{Edge-preserving Filters}

Traditional pyramid schemes, like the Laplacian pyramid decomposition or Toet's [14] contrast pyramid decomposition use linear (Gaussian) filters to obtain the approximation images described in 2.3. Even with a small kernel, they introduce halo artifacts into the images by blurring over sharp edges. This is because the filter is applied iteratively, which at higher levels is equivalent to a large filter kernel.

To prevent halo artifacts, edge-preserving filters have become popular, notably in the tone-mapping community, the most widelyused being the bilateral filter [15].

The bilateral filter performs well to separate an image into a base-layer, containing large scale variations, and a detail-layer capturing small scale variations such as texture. It is, however, less suited for progressive coarsening of images [2].

Farbman et al. [2] therefore describe an approach based on the weighted least squares optimization framework (WLS), obtaining a decomposition of a base-layer and detail-layers of different scales. Edge-preserved smoothing tries to find an image $u$ that simultaneously is as close as possible to the input image $g$ and as smooth as possible along significant gradients in $g$. Formally:

$$
u=W_{\lambda}(g)=\left(I+\lambda L_{g}\right)^{-1} g
$$

where $L_{g}=D_{x}^{T} A_{x} D_{x}+D_{y}^{T} A_{y} D_{y}$ with $D_{x}$ and $D_{y}$ being difference operators. $A_{x}$ and $A_{y}$ contain smoothness weights that depend on $g$. By increasing $\lambda$, the result becomes progressively smoother [2].

When using edge-preserving filters, no downscaling of the smoothed images should generally be performed as this would introduce aliasing artifacts because the images are not band-limited. As opposed to the wavelet approach, this representation is overcomplete.

\section{NIR INFORMATION FOR HAZE REMOVAL}

To fuse visible and NIR images, we start by transforming the visible RGB image into a luminance-chrominance color space. We obtain a one channel NIR image containing intensity data [1].

Let $I_{0}$ denote an image (visible or NIR), the extracted luminance of the visible image is denoted as $V_{0}$ and the NIR image as $N_{0}$. Both are the input for the image fusion algorithm. Its output is the fused luminance image $F_{0}$. 
The chrominance information of the visible image is not used in the fusion algorithm but simply recombined with the fused image $F_{0}$. This approach does not decrease perceived image quality because the human visual system is less sensitive to color information at high frequencies, a characteristic often exploited in image compression. However, more sophisticated color processing might improve the result (see Fig. 3, right).

We transform the visible and NIR images into their multiresolution representation, proceeding in two steps. First, we obtain approxiation images by using the WLS filter $(W)$ described by Farbman et al. [2].

$$
I_{k+1}^{a}=W_{\lambda_{0} c^{k}}\left(I_{0}\right)
$$

where the parameter $\lambda=\lambda_{0} c^{k}$ controls the coarseness of the approximation image at layer $k+1$. $\lambda_{0}$ expresses the coarseness of the first approximation image, the subsequent images are coarser by a multiple of $c$. We chose in our experiments $\lambda_{0}=0.1, c=2$ and a total number of levels of $n=6$.

Second, we obtain the detail images. But instead of using progressively coarser detail layers by substracting subsequent approximation images [2], we use Toet's [14] approach to obtain detail layers that measure contrast at progressively coarser scales, roughly analogue to Weber's contrast in a center-surround setting. Toet defines that the surround of a given pixel $I_{k-1}^{a}$ as the luminance of the same pixel in its blurred image $I_{k}^{a}$. Formally, this becomes:

$$
I_{k}^{d}=\frac{I_{k-1}^{a}-I_{k}^{a}}{I_{k}^{a}}
$$

The $n$ contrast images and the approximation image of level $n$ represent the multiresolution representation, see Fig. 2. The synthesis transform, i.e. obtaining the original image back form the multiresolution representation, is extremely simple. Indeed,

$$
I_{0}=I_{n}^{a} \prod_{k=1}^{n}\left(I_{k}^{d}+1\right)=I_{n}^{a} \frac{I_{n-1}^{a}}{I_{n}^{a}} \frac{I_{n-2}^{a}}{I_{n-1}^{a}} \cdots \frac{I_{1}^{a}}{I_{2}^{a}} \frac{I_{0}}{I_{1}^{a}}
$$

The fusion criterion is based on the following observations: The NIR image has a higher contrast in the presence of haze, therefore, we take the maximum of the visible $V_{k}^{d}$ and the NIR $N_{k}^{d}$ contrast images at each level $k$. The visible approximation image $V_{n}^{a}$ contains the low frequency luminance information as perceived by the

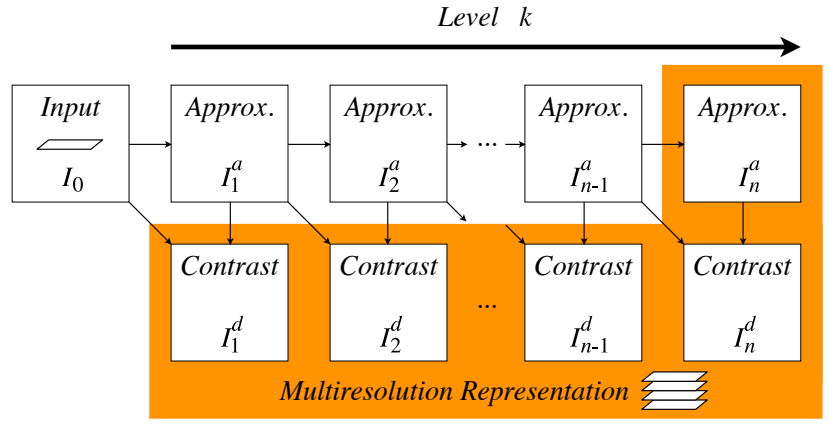

Fig. 2. n-level multiresolution analysis as defined by Toet [14]. For an input image $I_{0}$, the intermediary approximation images $I_{k}^{a}$ are obtained first. The detail images $I_{k}^{d}$, obtained in the second step, form with the approximation image $I_{n}^{a}$ the multiresolution representation.

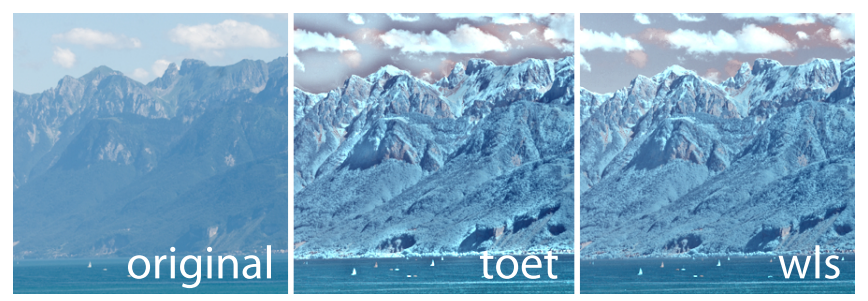

Fig. 3. Comparison of the original image, Toet's original approach and our improved approach using WLS filters. Note the halo artifacts that disappear by using edge-preserving filters.

human observer, the NIR approximation $N_{n}^{a}$ is thus discarded. The fused image is obtained by the synthesis transform:

$$
F_{0}=V_{n}^{a} \prod_{k=1}^{n}\left(\max \left(V_{k}^{d}, N_{k}^{d}\right)+1\right)
$$

\section{RESULTS AND COMPARISONS}

We compare the performance of our dehazing approach with different methods ${ }^{1}$. Using Toet's original algorithm, shown in Fig. 3, the contrast in hazy areas is significantly increased and additional hidden details appear. However, halo artifacts are present at sharp edges where the luminance of the NIR and visible image are significantly different. By making use of the WLS filters, these artifacts disappear.

Comparing with the state-of-the-art dehazing technique [8], we can see in Fig. 4 that our results contain more details and texture. The results of He et al. and Fattal, however, yield more realistic colors, as their approach is based on the haze formation equation while we only manipulate the luminance channel. He et al.'s technique on the other hand yiels some halo artifacts in the left image of Fig. 4.

Indeed, our technique is comparable to the approach of Fattal et al. [16] for multiresolution shape and detail enhancement from multi-light image collections. In our case, we have not differently lit images, but images from different parts of the spectrum.

\section{CONCLUSIONS}

We present an algorithm that successfully improves the contrast of haze-degraded color images. Our approach is based on multiresolution image fusion of visible and NIR information. We have combined a state-of-the-art edge-preserving smoothing filter with a very simple multiresolution decomposition based on contrast images.

Our method improves any scattering degradation following Rayleigh's law. Compared to local contrast enhancement methods, our approach does not need a heuristic to detect haze as NIR images are intrinsically haze-free. As the high frequency content of NIR images is otherwise very similar to the visible, our algorithm can be applied to any image, regardless of the actual presence of haze, without introducing artifacts.

Moreover, the additional NIR information allows for dehazing without requiring a scattering model. Therefore no assumptions are needed in order to generate a correct depth map. No heuristic to detect the airlight, an error prone task, is necessary.

\footnotetext{
${ }^{1}$ additional are available at ivrg.epfl.ch/supplementary_material/SFS_ICIP09/
} 


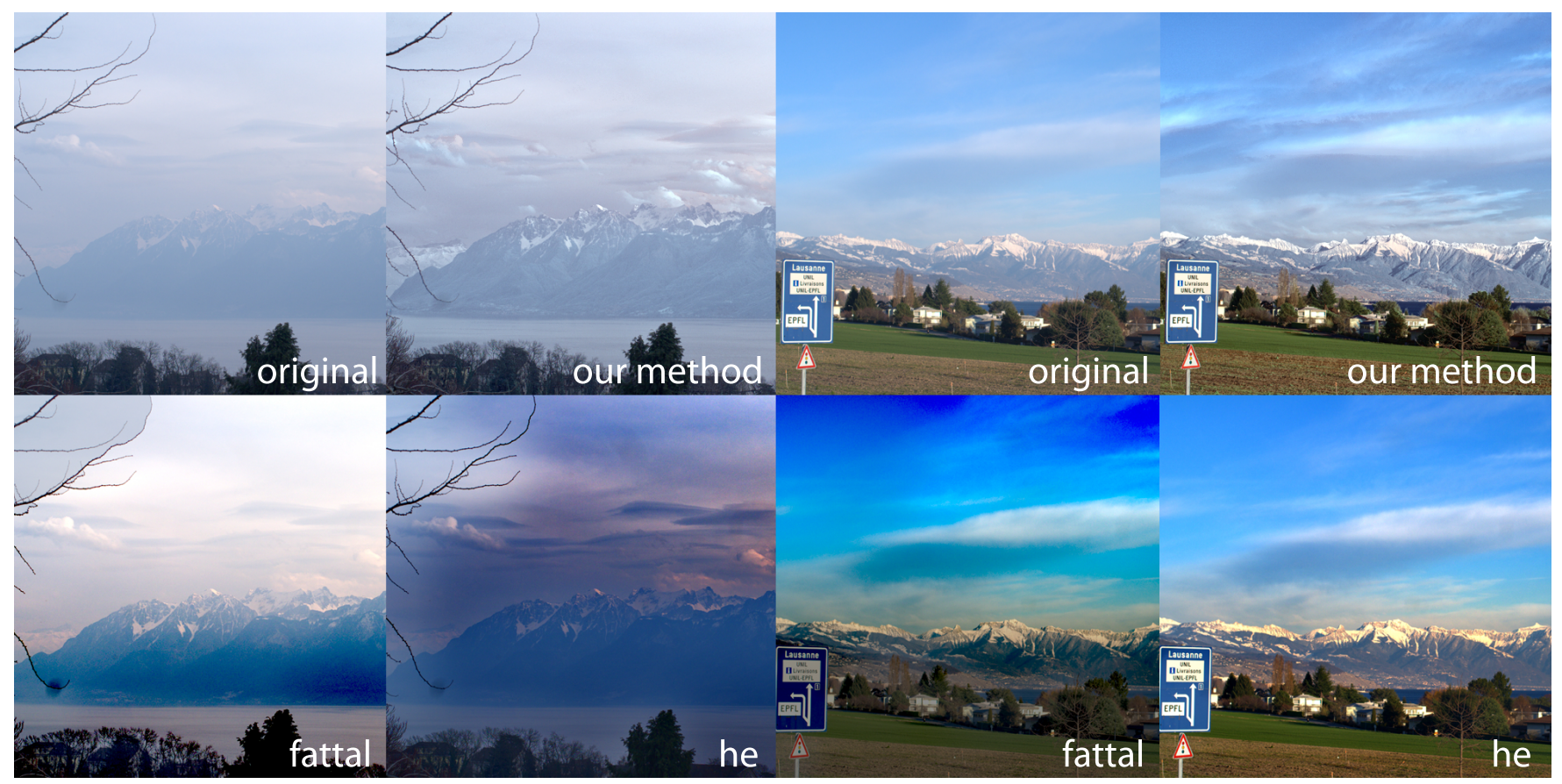

Fig. 4. Comparison of the original image, He et al.'s dehazing approach (Courtesy of K. He), Fattal's dehazing approach (Courtesy of Prof. R. Fattal) and our multiresolution fusion using WLS filters.

As we are only working with the luminance channel, colors might appear unrealistic in cases of extreme luminance changes (Fig. 3 ). We want to investigate in the future how our images can be improved by additional color processing.

\section{REFERENCES}

[1] C. Fredembach and S. Süsstrunk, "Colouring the near infrared," IS\&T 16th Color Imaging Conference, pp. 176-182, 2008.

[2] Z. Farbman, R. Fattal, D. Lischinski, and R. Szeliski, "Edgepreserving decompositions for multi-scale tone and detail manipulation," International Conference on Computer Graphics and Interactive Techniques, pp. 1-10, 2008.

[3] M.L. Groszek and J.P. Allebach, "Haze removal for image enhancement," Proceedings of the SPIE, vol. 5678, pp. 254265,2005

[4] Y.Y. Schechner, S.G. Narasimhan, and S.K. Nayar, "Instant dehazing of images using polarization," IEEE Conference on Computer Vision and Pattern Recognition, vol. 1, pp. 325-332, 2001.

[5] S.G. Narasimhan and S.K. Nayar, "Contrast restoration of weather degraded images," IEEE Transactions on Pattern Analysis and Machine Intelligence, vol. 25, no. 6, pp. 713-724, 2003.

[6] J. Kopf, B. Neubert, B. Chen, M. Cohen, D. Cohen-Or, O. Deussen, M. Uyttendaele, and D. Lischinski, "Deep photo: model-based photograph enhancement and viewing," International Conference on Computer Graphics and Interactive Techniques, pp. 1-10, 2008.
[7] S.G. Narasimhan and S.K. Nayar, "Interactive (de) weathering of an image using physical models," IEEE Workshop on Color and Photometric Methods in Computer Vision, 2003.

[8] K. He, J. Sun, and X. Tang, "Single image haze removal using dark channel prior," IEEE Conference on Computer Vision and Pattern Recognition, pp. 1957-1963, 2009.

[9] R.T. Tan, "Visibility in bad weather from a single image," IEEE Conference on Computer Vision and Pattern Recognition, pp. 1-8, 2008.

[10] R. Fattal, "Single image dehazing," International Conference on Computer Graphics and Interactive Techniques, pp. 1-9, 2008.

[11] Y.M. Lu, C. Fredembach, M. Vetterli, and S. Süsstrunk, "Designing color filter arrays for the joint capture of visible and near-infrared images," IEEE International Conference on Image Processing, 2009.

[12] A.A. Goshtasby and S. Nikolov, "Image fusion: Advances in the state of the art," Information Fusion, vol. 8, no. 2, pp. 114118, 2007.

[13] R.S. Blum and Z. Liu, "Multi-sensor image fusion and its applications," CRC Press, 2006.

[14] A. Toet, "Hierarchical image fusion," Machine Vision and Applications, vol. 3, no. 1, pp. 1-11, 1990.

[15] S. Paris, P. Kornprobst, J. Tumblin, and F. Durand, "A gentle introduction to bilateral filtering and its applications," International Conference on Computer Graphics and Interactive Techniques, 2007.

[16] R. Fattal, M. Agrawala, and S. Rusinkiewicz, "Multiscale shape and detail enhancement from multi-light image collections," International Conference on Computer Graphics and Interactive Techniques, vol. 26, no. 3, 2007. 